\title{
Consommation de tabac : Facteur de risque pour la consommation de drogues
}

\begin{abstract}
This article aims to highlight the role of smoking as a risk factor for the use of drug among adolescents. Since the latter increasingly being perceived by adolescents as frustrating social codes transgression, there is the desire in it to play with risk, while sucking also to reach through this act to a state of wholeness and self-assertion, from there, his identity is built, his personality is confirmed; a personality rather suspicious towards adults.

The objective of this research is to analyze and discuss adolescence as a period of risk which involves adolescents in several behavioral problems including smoking and drugs taking which are the most important. From this synthesis the researcher has emphasized on the process of addiction and tobacco use as one of the factors of illegal drugs consumption. In this article we have mentioned the most important challenges encountered by the United Nations, and nongovernmental organizations in facing the phenomenon of smoking which is not only a public health problem and all associated ones, but a risk factor for the involvement of adolescents in more complicated problems such as addiction to illicit drugs.

Keys words: tobacco use - drug Use - risks factorsadolescents - psychosocial factors.
\end{abstract}

\section{Farida GUEMAZ \\ Fayza DJENIDI}

Faculté des Sciences Humaines et Sociales Université Sétif 2

(Algérie)

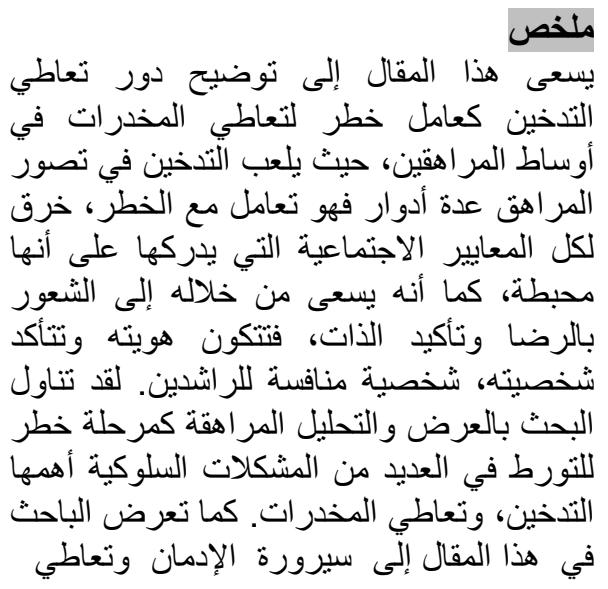

\section{Introduction}

Le tabac représente un des plus importants fléaux de santé publique qui ne peut qu'interpeller davantage les consciences, ainsi que l'une des plus grandes menaces auxquelles les institutions mondiales de la santé doivent faire face en ces débuts de siècle, devant l'ampleur du phénomène et sa propagation fulgurante et rapide, ces dernières se trouvent confrontées à des chiffres alarmants qui ne peuvent que les 
التبغ كعامل من عو امل الخطر لتعاطي المخدرات

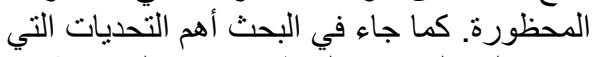

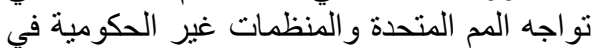
التعامل مع ظاهرة تعاطي التبغ ليس باعتبار ها

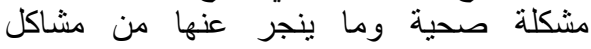

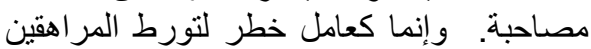

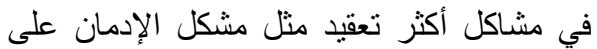

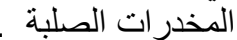

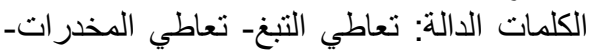

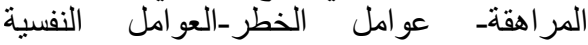
الاجنماعية

impliquer à juste titre, profondément ancrés dans les mentalités et les croyances sociales des personnes et encourager par une publicité attractive appuyée par une industrie de tabac imbattable, les comportements tabagiques qui se caractérisent par un haut degré de dépendance sont à l'heure actuelle une épidémie de grande envergure, puisque 'l'Organisation Mondiale de la Santé en tant que première institution se préoccupant de la santé des individus, estime que le tabac tue 6 personnes par minute dans le monde, et à l'horizon 2025, le nombre de décès imputables au tabac serait de 10 millions par an dont 7 millions dans les pays en

développement (WHO, 1998). Tandis que dans les pays industrialisés on constate une régression de cette épidémie, les constatations faites dans ce sens révèlent une hausse de la consommation de tabac dans les pays en voie de développement En effet et d'après Bartecchi et al. (1995)., suite aux mesures rigoureuses de contrôle de la publicité sur le tabac dans la plupart des pays industrialisés, les firmes de production de tabac se sont orientées vers les pays du tiers monde, notamment le continent africain qui constitue un terrain de prédilection pour promouvoir la consommation de tabac cette épidémie n'épargne pas les jeunes et les moins jeunes qui s'initient précocement aux conduites tabagiques, et deviennent un public potentiel pour la promotion d'un telle production,(Warren et al. (2008), avancent que selon, l'enquête mondiale sur le tabagisme chez les jeunes écoliers de 13 à 15 ans «GYTS» initiée par l'OMS, UNICEF et CDC Atlanta, qui a été conduite dans 140 pays entre 1999 et 2007, a montré que le pourcentage des jeunes consommant des produits du tabac allait d'un maximum de $30 \%$ à un minimum d'environ $4,9 \%{ }^{9}$

la consommation du tabac est relatif à un âge et à une préférence spécifiques, puisqu'il est particulièrement la première substance toxique que les adolescents aiment tester, bien que l'alcool reste le produit le plus consommée, mais le plus inquiétant est le fait que, chez les adolescents, la consommation de cigarettes s'accompagne souvent d'alcool et d'autres substances illicites, et il est également relatif à un milieu bien déterminé, car d'après les même résultats de l'enquête mondiale Global Youth Tobacco Survey (GYTS) sur le tabagisme chez les jeunes, publiés par l'OMS ont estimé qu'en Afrique $20 \%$ des 13-15 ans fument en milieu scolaire.,iI faut dire que dans le monde, entre 13 et 15 ans, 1 adolescent sur 5 fume du tabac. 50\%de ceux qui ont commencé à cet âge continuent après 20 ans, ceci constitue un danger pour la santé de la population mondiale étant donné que le tabagisme, surtout commencé jeune, est source ultérieure de multiples problèmes de santé 23

Il semblerait que l'interaction complexe entre plusieurs types de facteurs est à l'origine de la consommation du tabac, chez les jeunes, et si parmi ces facteurs certains sont modifiables d'autres par contre présentent des formes sur lesquelles il est difficile d'intervenir, le tabac et lui-même considéré comme un facteur de risque 
potentiel pour la consommation des autres produits, étant donné que le développement de la dépendance à tout produit induisant une addiction est directement lié à un processus d'apprentissage avant et pendant l'adolescence, et parallèlement lié à une phase de curiosité ,de prises de risque et de défi durant laquelle le jeune adolescent recherche de nouvelles expériences associant souvent une certaine résistance aux règles établies.

Pour les jeunes adolescents le recours au tabac traduit une volonté de jouer avec le risque tout en éprouvant la satisfaction de transgresser les codes sociaux, le franchissement de la limite ajoute sa saveur aux sensations recherchées, de plus l'accessibilité croissante au tabac rend la tentation difficile à repousser, une quête d'identité se construit là , en opposition aux adultes, groupe de pairs et pressions sociales peuvent pousser davantage le jeune à fumer d'où l'importance de développer des facteurs de résistance et prévenir des conduites addictives, ainsi que d'encourager l'esprit critique chez le jeune fumeur et lui permettre d'identifier les risques encourus et également discerner entre tentation, imitation, et recherche de sensations

L'accessibilité et l'acceptabilité, du tabac dans nos sociétés deux autres paramètres pertinents, ont tendance à banaliser l'acte de fumer chez le jeune, cependant le passage d'un produit licite à un autre illicite peut se faire facilement, d'ailleurs, tabac, alcool et drogues sont tous des produits substitutifs l'un à l'autre car sous l'emprise des tensions générées par la période de l'adolescence, période critique ou la demande se fait de plus en plus pressante, l'adolescent est contraint de trouver d'autres substances plus fortes pour se libérer des contraintes d'identité et élargir les possibilités d'action sur le monde et rompre ses inhibitions pour se rendre plus sensible à une quelconque ambiance .

Au centre de cette problématique les questionnements suivants :

-La consommation de tabac peut-elle être un facteur de risque pour la consommation de drogues?

-Si c'est le cas doit-on décrypter très tôt les représentations des risques encourus en cas de consommation de tabac chez le jeune adolescent afin de prévenir d'autres pratiques addictives?

\section{1-Période de l'adolescence}

L'adolescence est une période propice à de nombreux remaniements, tant sur le plan interne que sur le plan interpersonnel, c'est une période charnière entre l'enfance et l'âge adulte ou se manifestent des changements physiques, psychologiques et éventuellement de nouveaux comportements sociaux, elle correspond non seulement à un processus tumultueux de maturation mais c'est aussi à une étape ou se rejoue le processus de séparation et d'individuation, ce cheminement vers l'autonomie amène la plus part des adolescents à vouloir dépasser certaines limites et à expérimenter de nouveaux comportements et à vivre de grands changements, des comportement plutôt de prise de risque pouvant avoir des conséquences négatives, puisque considérée comme une période de difficultés et de riches découvertes. 


\section{a-Adolescence phase d'expérimentation}

Nicholson,et al, (2000), nous confirment par le biais de nombreuses études que l'initiation et l'expérimentation des conduites à risque restent particulièrement liées à cette période évolutive, certaines conduites à risques visent à satisfaire la curiosité , l'expérimentation de la vie et la recherche de sensations, d'autres conduites réalisent le besoin de contester et de remettre en question les limites sociales et morales.$^{17}$

C'est aussi une période au cours de laquelle l'adolescent tout en voulant exprimer ses désirs et affirmer son nouveau statut se détache progressivement de ses parents qui ne peuvent plus être les figures principales comme au temps de l'enfance et choisis d'autres figures d'identification, et d'autres relations, au sein desquelles il peut assouvir son désir pour expérimenter tout comportement ou substance sans aucun control, dans l'intention de résister aux règles établis et s'émanciper celui-ci s'imprègne facilement des conduites et des agissements de ses pairs en recherchant constamment leur reconnaissance et transgressant en leur compagnie toutes les barrières, en effet c'est essentiellement pendant cette, période d'intégration dans le cercle des pairs et de prise de distance vis-à-vis des parents, que se font les premières expériences telles que l'initiation à la consommation du tabac ainsi qu'à la déscolarisation ou tout autre comportement négatif.

Varescon Isabelle ,(2012),stipule queL'adolescent emporté dans les conduites à risque est d'abord dans une souffrance affective, même si sa condition sociale et son sexe ajoutent une dimension propre. Seule son histoire personnelle et la configuration sociale et affective où il s'insère sont susceptibles d'éclairer le sens de comportements, certains de ces comportements s'inscrivent dans la durée (toxicomanies, troubles alimentaires, scarifications, alcoolisation, repli sur le jeu vidéo ou le web, errance...), d'autres en revanche relèvent d'un acte unique lié aux circonstances (tentatives de suicide, fugue, défi relevé, etc.) ${ }^{16}$

Lafaye De Micheaux, (2008),ajoute de son coté que cette étape du développement, qui mobilise fortement le système d'exploration, va donc être une période où les expérimentations seront les plus fréquentes, dont notamment celles autour de l'usage de substances psychoactives. Alors que bon nombre d'adolescents auront recours à une pratique addictive, seule une minorité s'engagera à plus long terme dans des conduites d'abus ou de dépendance. ${ }^{10}$

\section{b-Période propice à l'apparition des conduites à risque}

En plus d'être une phase transitoire et un passage critique ou se rejoue un bon nombre de remaniements tant sur le plan affectif ou relationnel, l'adolescence est aussi une période favorable à l'émergence des conduites à risques et des conduites addictives comme le tabagisme par lesquelles le tabac commence, comme un facteur social encore valorisé par notre culture et par les pairs mais par la suite il enferme nos jeunes dans un piège dont il n'est pas facile de sortir tant cette dépendance est forte.

Puisque, d'après Kerjean (2005), Le problème avec un produit comme le tabac est la puissance de la dépendance physique et psychologique qu'il induit, cette dépendance va entraîner très rapidement une consommation régulière et une perte de contrôle sur la 
liberté de ne pas fumer. L'arrêt du tabac sera alors plus compliqué que prévu, ${ }^{8}$ ' est pourquoi les pouvoirs publics et les professionnels de santé sont sensibilisés a juste titre, le souci majeur de cette mobilisation est l'inquiétante prolifération des pratiques de consommation de substances psychoactives parmi les jeunes générations, les principales raisons de cette préoccupation est que l'adolescent est un être vulnérable et un être en devenir qui doit acquérir bon nombre de compétences émotionnelles et sociales mais aussi scolaires et le risque d'engagement dans l'addiction d'un tel ou autre produit nocif ne saurait que compromettre ce processus indispensable pour l'accomplissent final du développement de cette période.

D'après, Beck \& Legleye, (2009) c'est au cours de l'adolescence que s'opère une diffusion rapide des usages de produits psychoactifs jusqu'au début de l'âge adulte, avec des contrastes suivant les produits, niveaux élevés pour le tabac et dans une moindre mesure l'alcool, plus faibles pour le cannabis et surtout pour les autres substances illicites Cependant, une substance psychoactive est rarement consommée seule, ce qui incite à prendre en compte, en plus du problème des polytoxicomanies, l'ordre dans lequel différentes substances sont susceptibles d'être initiées afin de déterminer des priorités en termes de prévention. ${ }^{11}$

Avant d'aborder la problématique de la succession de la consommation de plusieurs produits, il est nécessaire de bien cerner la problématique autour de l'usage du tabac chez les adolescents donc il faudrait d'abord comprendre le phénomène de dépendance qui répond aux même processus pharmacologiques et comportementaux que celui de la dépendance à l'égard des drogues comme l'héroïne et la cocaïne.

\section{c-Le phénomène de dépendance}

le tabagisme est classé par l'OMS parmi les toxicomanies (syndrome de dépendance lié à l'usage du tabac la dépendance à une substance psychoactive se définit par un mode d'utilisation inapproprié d'une substance, entraînant une détresse ou un dysfonctionnement cliniquement significatif. ${ }^{6}$

Les résultats des études menées dans ce sens ont démontrées que le tabagisme est un comportement entretenu et renforcé par une dépendance,qui suit un processus bien déterminé étant donné qu' après la consommation d'un produit tabagique, l'absorption de la nicotine en raison de ses propriétés biologiques déclenche des modifications qui sont responsables de la dépendance. Le tabac rend le fumeur dépendant de deux façons différentes selon la façon dont la dépendance s'exprime au sevrage. Il existe une dépendance qui est liée aux effets psychoactifs de la nicotine (plaisir, stimulation intellectuelle s, action antidépressive.), c'est la dépendance psychique, ou une dépendance qui est liée au manque de nicotine dite dépendance physique. ${ }^{5}$

\section{d-Aspects biologiques de La consommation tabac}

La diversité et la qualité des études en biologie et en médecine fournissent des évidences tellement importantes sur l'aspect biologique de la consommation qu'on ne doit pas écarter mais plutôt le considérer au coté de l'aspect psychosocial comme déterminant, pour comprendre le phénomène de la dépendance aux substances 
psychoactives, on ne peut donc plus aborder ce dernier que dans un modèle multifactoriel. $^{3}$

\section{*Le parcours de la Substance Psycho Active dans le corps}

Pour agir, la SPA doit d'abord se rendre à son site d'action principal, le cerveau, elle est l'objet de phénomènes biologiques complexes, le mode d'administration de la substance déterminera le temps que la SPA prendra dans le corps, trois voies sont essentiellement reconnues, on retrouve dans l'ordre : la voie intraveineuse, la voie intrapulmonaire (fumé), la voie intra nasale (prisé ou « sniffé »), et la voie orale,

La voie intraveineuse est la voie la plus rapide et la plus efficace, injectée directement dans la circulation sanguine, la SPA ne prend que quelques secondes à se rendre au cerveau. A l'opposé, la voie orale est la plus lente et la moins efficace car une partie de la substance sera détruite par les enzymes et l'acidité de l'estomac, l'effet d'une SPA absorbée par voie orale prend généralement entre 15 et 60 minutes avant de se faire sentir. ${ }^{3}$

Pour bien cerner la problématique autour de La dépendance qui correspond à une perturbation du fonctionnement cérébral suscitée par l'utilisation de substances psychoactives.et modifiant les processus cérébraux normaux de la perception, des émotions et des motivations.il faut d'abord connaitre le fonctionnement normal du cerveau pour apprécier son dysfonctionnement, un dysfonctionnement qui peut être à l'origine de symptômes comportementaux hautement complexes. Une grande variété de maladies et de traumatismes peuvent affecter le cerveau, dans tous les cas, le comportement qui en résulte est reconnu comme partie intégrante de l'affection c'est pareil pour la dépendance, l'entité comportementale résultante est complexe, mais essentiellement liée aux effets à court terme et à long terme des substances sur le cerveau. $^{19}$

toujours dans la même optique toute substance absorbée par un adolescent ne produit pas le même effet que celui produit sur un cerveau d'une personne adulte,connu comme étant une substance extrêmement neurotoxique, la nicotine peut avoir des effets épigéniques surtout au niveau des neurones immatures. C'est donc la biologie des adolescents dans les périodes précoces et l'immaturité de leurs cerveaux qui prédisposeraient les substances à avoir des effets néfastes, neurotoxiques, pathologiques ou comportementaux à long terme, car il faut tout de même admettre que la vulnérabilité de l'adolescent n'est uniquement un artefact socio circonstanciel sa vulnérabilité est plutôt du à l'état de son cerveau et à l'influence de la substance sur celui-ci.

\section{2-Facteurs de risque}

C'est un ensemble de facteurs endogènes et exogènes entremêlés qui seraient à l'origine de la dépendance et qui sont le plus souvent révélateurs d'un état vulnérable, soit sur le plan psychologique ou social, des facteurs liés à la personnalité et à certaines caractéristiques de l'adolescence peuvent contribuer au début de la consommation de tabac. 
Pour les adolescents, fumer est souvent un rite de passage à l'âge adulte. La tendance à prendre des risques, la curiosité pour de nouvelles expériences ou encore le besoin de transgresser des règles favorisent notamment l'expérimentation de la cigarette. Des troubles psychologiques comme l'anxiété et la dépression peuvent encore favoriser la consommation de cigarettes une forte estime de soi et la connaissance des dangers de la consommation jouent également un rôle dans le début de consommation, mais qui peut aussi diminuer les risques de commencer à fumer. ${ }^{20}$

Il existe trois grandes catégories de facteurs de risque (liés au produit, individuels et environnementaux) dont les interactions sont primordiales à prendre en compte, puisque responsables des différents modes de consommation de substances psychoactives.

D'après le rapport du Surgeon General des États-Unis publié en 1994 sur la prévention du tabac, il existerait cinq étapes avant qu'un adolescent ne devienne dépendant physiquement du tabac. Durant la première étape, ou étape préparatoire, les croyances et les attitudes au sujet de l'utilité du tabagisme se développent. Le tabagisme peut être vu, entre autres, comme une façon de paraître mature, indépendant, comme un moyen de faire face au stress ou encore d'établir des liens avec un groupe d'amis. La deuxième étape est la période d'essai, celle durant laquelle le jeune fume ses premières cigarettes, habituellement avec des amis. L'expérimentation, la troisième étape, est la période durant laquelle le jeune fume de façon répétée, mais irrégulière, lors de fêtes ou avec ses meilleurs amis alors qu'au cours de la quatrième étape, il fume chaque semaine et qu'ainsi l'usage du tabac est qualifié de régulier. Finalement, le jeune deviendrait dépendant physiquement au tabac à la cinquième étape. ${ }^{2}$

\section{a-Facteurs de risque liés au produit}

Il existe plusieurs facteurs liés au produit ,qui sont également liés au risque de dépendance et aux risques de complications somatiques, psychologiques et sociales, si on prend en compte les trois substances psychoactives les plus addictives qui sont le tabac, l'héroïne et l'alcool, les facteurs qui sont à l'origine de la consommation sont d'ordre sociodémographique, tel que le passage de l'école élémentaire à l'école secondaire ou l'initiation au tabagisme serait un moment à haut risque, ainsi que le sexe qui serait un indice important pour le repérage de la prévalence de la consommation parmi les adolescents et les adolescentes,

Ce sont aussi des facteurs socio-économiques tels que le niveau de vie familiale et le bas niveau d'éducation des parents qui seraient des facteurs de risque prédictifs de l'initiation au tabagisme et sont associé à un taux de tabagisme plus élevé chez les jeunes.

\section{b. Facteurs de risque individuels}

Quand aux facteurs individuels ils sont plutôt liés au bagage génétique des personnes, certaines seraient moins prédisposés que d'autres à devenir dépendantes du tabac, des hypothèses tel que par le rythme auquel la nicotine se métabolise ou encore la différence de la neurotransmission dopaminergique de ces personnes seraient probablement pris en considération comme explication plausible à l'avenir. 
Cela semble être le cas pour des substances qui inhiberaient l'action d'un enzyme, le CYP 2A6, responsable de métaboliser de $70 \%$ à $80 \%$ de la nicotine en cotinine, ces différences dans le bagage génétique des individus pourraient aussi expliquer ce qu'une recension des écrits au sujet des premiers épisodes d'usage du tabac chez les jeunes révèle, à savoir que les adolescents qui deviennent des fumeurs réguliers rapportent moins d'effets désagréables lors de leurs premiers essais avec le tabac. ${ }^{2}$

Autres facteurs, les facteurs psychologiques,. ce sont les caractéristiques tempéramentales et traits de personnalité, La cigarette donne une autre image de soi, une valorisation qui donne l'impression au fumeur d'être intégré dans le monde actuel (raffiné, viril .....). ici le besoin de fumer répond à un état de fuite d'abord face à son anxiété, les gestes rituels aident le fumeur à supprimer son anxiété momentanément et il lui semble qu'il la domine et ensuite à un vide que la cigarette vient combler, là le malaise existentiel est indéterminé, Et enfin à un manque que le fumeur n'arrive pas à définir et à se le préciser en lui même. Et probablement à un échec, là le fumeur se sent incapable à faire face aux difficultés. ${ }^{13}$

Il semblerait aussi qu'en relation avec les facteurs de personnalité, la recherche de sensations puisse aussi intervenir comme un ensemble d'attitudes, de conduites et de comportements pouvant également expliquer la nature de cette recherche de sensation, d'après Zuckerman, 1984 et selon la théorie de la recherche de sensations qui postule que les amateurs de sensations, quand ils se trouvent dans un état de non-stimulation avec une activité catécholaminergique faible, recherchent des substances ou comportements qui relèvent cette activité de plus, il semblerait que cette dimension de personnalité module les réponses subjectives et psychophysiologiques aux drogues. Hutchinson et ses collaborateurs (1999) ont montré qu'elle influence les effets stimulants de l'amphétamine. Selon les auteurs, « la recherche de sensations correspond à une haute sensibilité aux effets d'une substance psychostimulante. ${ }^{22}$

\section{c-Facteurs de risque environnementaux}

Ces facteurs ne peuvent être pris isolément et ne peuvent pas être considérés comme un élément de causalité linéaire parmi les principaux facteurs de risque environnementaux les facteurs familiaux.Pour Chabrol, (1995)., la famille semble jouer un rôle important dans le déterminisme ou la prévention de l'usage de substances à l'adolescence. On retrouve régulièrement et par ordre de gravité croissante : des conflits familiaux ou une tension relationnelle majeure, des habitudes de consommation abusive ou excessive chez les parents, des antécédents de deuils familiaux non résolus, une cécité des membres de la famille envers des comportements chez le jeune qui devraient susciter l'inquiétude ou, à l'inverse, une sollicitation anxieuse exacerbée chez l'un des parents; à l'inverse, la proximité entre parents et enfants, l'affection et le support parental apparaissent comme des facteurs de protection.

Il ya également les facteurs culturels et sociaux comme la perte des repères sociaux que sont la précarité, le chômage, certains quartiers défavorisés ainsi que tous les processus conduisant à l'exclusion ou à la marginalisation du jeune ont un effet favorisant et incitatif sur la consommation addictive de produits. Le plus important reste de loin l'échec ou la rupture de la scolarité ainsi que le rôle des pairs. Cependant, 
si à l'évidence la pression des pairs est un facteur d'abus de substances, elle peut être également un possible facteur d'abstinence. En effet, il existerait une relation directe entre une pression croisée des pairs et un usage de drogues, ce qui ferait que plus l'acceptation de drogues est basse, moins fréquent serait son usage. parmi les facteurs environnementaux il y'aussi acceptabilité et accessibilité du tabac. ${ }^{10}$

Car L'acceptabilité et l'accessibilité sont aussi deux facteurs non négligeables dans la prolifération de la consommation du tabac dans les milieux des adolescents et influenceraient considérablement le comportement tabagique chez ces derniers, puisque l'absence de l'instauration de lois relatifs à l'interdiction de vente de tabac aux écoliers et aux mineurs n'a pas et ne sera probablement pas promulgué à l'encontre de ce genres d'activités commerciales, dans le souci majeur est la protection de la santé de l'enfant et l'adolescent, l'accès facile au tabac par le biais du faible prix des produits du tabac est aussi une motivation pour que le consommateur jeune en abuse sans aucune retenue, pareillement pour l'identification de ce dernier au monde des adultes par l'intermédiaire par exemple d'acteurs ou de chanteurs faisant usage du tabac et véhiculant un comportement de prestige et de distinction auquel l'adolescent ne peut être que sensible, et par lequel celui-ci affirme davantage et de plus en plus son intégration dans la sphère des adultes.

\section{3-Le tabac en tant que facteur de risque chez les jeunes}

Si précédemment on a évoqué un ensemble de facteurs pouvant être à l'origine de la consommation de tabac et d'autres substances psychoactives, le tabac en lui-même est un facteur de risque éminent pour d'autres pathologies, il est le premier et le principal produit psychoactif consommé de façon quotidienne, quel que soit le sexe, même si les filles l'expérimentent plus tardivement. L'installation dans un usage quotidien survient souvent pendant les « années de collège, si le tabac est le premier produit psychoactif licite à être consommé par les jeunes. Le risque principal est le développement rapide d'une addiction à la nicotine, substance reconnue comme étant très addictogène ${ }^{4}$ en plus d'être considéré comme un risque potentiel lié à l'utilisation d'autres substances psychoactives c'est aussi un facteur de risque connu de plusieurs pathologies. les effets à court terme du tabagisme s'observent chez les jeunes au niveau de l'appareil respiratoire (infections dont la tuberculose, baisse de la fonction ventilatoire, symptômes tels que les sifflements, la toux, le crachat.), les effets sur le système buccodentaire (caries, gingivites...), parmi les fumeurs dépendants, les manifestations sont plutôt liés aux phénomènes d'addiction, le tabac diminue la fertilité et, en cas de grossesse, augmente les risques de fausses couches et d'accouchement prématuré 5 .

Sur un plan psychique, la nicotine développe une dépendance semblable à celles des drogues dures. Elle agit sur le circuit dopaminergique de la récompense au même titre que la cocaïne, l'héroïne. ${ }^{14}$

\section{4-Les motivations et représentations associés à l'usage des produits psychoactifs}

Afin d'améliorer, in fine, l'efficacité des stratégies de prévention il faut déjà s'intéresser aux intentions des consommateurs de produits et à leurs raisons d'agir, en relation avec leur contexte de vie. 
Les rares études menées dans ce contexte relèvent la variété des motivations d'usage, d'un produit à l'autre mais aussi pour un même produit, selon Kuntsche et al.( 2006), ces motivations sont, rechercher du plaisir (motifs "hédoniques"), dans un esprit de partage et de convivialité (les drogues comme "lubrifiant social" ou comme élément festif); lutter contre l'anxiété et le stress, mieux dormir ou moins souffrir (motifs "autothérapeutiques"); surmonter un obstacle ou améliorer ses performances (fonction "stimulante" des drogues); rompre l'ennui ou passer le temps (motif de "distraction") ; oublier les contraintes et les difficultés ou s'oublier soi-même (recherche d'échappatoire). Quelques travaux anglo-saxons ont, par exemple, établi que les principaux motifs de consommation ont à voir avec le plaisir, l'accessibilité des produits et une représentation euphémisée des risques liés à l'usage tels que les travaux de(Akré et al. 2010; Amos et al. 2004; Menghrajani et al. 2005; Moore 2008) ${ }^{24}$

Il est claire que c'est de la petite enfance à la post-adolescence, que la prévention des conduites addictives doit être conduite avec le souci constant d'empêcher, de retarder ou de limiter les consommations, sans proposer de réponses. Elle doit être intégrer dans une politique de promotion globale de la santé et surtout être accompagné d'un travail de renforcement des compétences psychosociales des jeunes et de celles de leurs parents.la meilleure façon d'agir, et d'aider les jeunes à dire non, et les parents à ouvrir et maintenir le dialogue pour mieux cerner les difficultés de leurs enfants.

L'usage début par des rites avec les « copains » ou les membres de la famille, et bien que les premières cigarettes soient plutôt aversives et donnent souvent des nausées, les troubles désagréables disparaissent rapidement par l'installation d'une tolérance, et des effets psychoactifs positifs apparaissent avec sensation de plaisir, de détente, de stimulation intellectuelle, de réduction de l'appétit, de diminution de l'anxiété et de la timidité. Dès que la consommation devient quotidienne, cette dépendance psychologique est suivie d'une dépendance physique caractérisée par la sensation de manque et le besoin irrésistible de fumer (Lagrue, 2000). A dix-huit ans, un adolescent sur deux consomme du tabac et un sur quatre consomme déjà 10 cigarettes par jour et plus (Choquet et coll., 1995). ${ }^{25}$

Lagrue et coll., (2001) estime que chez un adolescent fumeur sur cinq, la résine de cannabis s'associe très tôt à la cigarette-tabac sous forme de «joint »; dans ce mélange les effets de chaque produit sont renforcés. ${ }^{12}$

\section{5-Diverses trajectoires possibles de consommation}

Il est possible de mettre en évidences la possibilité qu'il existe différentes logiques de consommation en matière de drogue ainsi que diverses trajectoires entretenues par les consommateurs, comme par exemple qui a fumé du tabac est plus susceptible de fumer du cannabis, et qui a éprouvé l'ivresse de l'alcool peut, si l'occasion s'en présente, avoir envie d'expérimenter l'ivresse que provoquent d'autres drogues. S'il est vrai que la consommation de substances telles l'héroïne ou la cocaïne passe très souvent par la consommation préalable de cannabis, elle passe aussi par la consommation d'autres substances, notamment le tabac et l'alcool. L'image des produits joue aussi un rôle essentiel, une personne ayant déjà sniffé de la cocaïne peut refuser de faire de même avec l'héroïne car ce produit est associé à un univers négatif, 
qui a pris l'habitude d'avaler des médicaments psychotropes prendra plus volontiers un ecstasy ou un LSD, qui a déjà " sniffé » une poudre, de la cocaïne par exemple, expérimentera plus facilement de l'héroïne, de l'amphétamine ou de la kétamine. Et il est certain qu'au-delà d'un certain nombre de produits, la " peur de la première fois » cède la place à l'excitation de vivre une nouvelle expérience de l'ivresse. ${ }^{12}$

L'hypothèse d'une relation entre la consommation du tabac comme un risque potentiel pour la consommation d'autres drogues, et qu'une substance licite est susceptible d'induire un usage de drogue illicites a été introduit dans les années 70 (Kandel, 1975; Kandel \& Faust, 1975). A partir de là une théorie a émergé en voulant mettre en évidence une relation d'enchainement entre les différentes consommations, c'est la (Gateway theory) ,ou la théorie de l'escalade qui suppose que la consommation de drogues dites douces conduit inexorablement à l'usage de drogues dures, ce fut Denise Kandel il y a maintenant plus de 30 qui a fait remarquer qu'il existait une trajectoire d'usage définie, par une séquence temporelle d'initiation des différentes substances et qui repose sur l'association statistiques entre les usages, le risque d'initiation des substances représenté selon Kandal serait en évolution constante et il a également un début et une fin, suivant une trajectoire d'usage appelé (Gateway sequences) ,pour être plus explicite, tout sujet ayant fait usage d'une quelconque substance et à risque d'en expérimenter une suivante selon la trajectoire dans laquelle il est rentré, de là Kandal, suggère que tabac et alcool peuvent être considérés comme portes d'entrée vers des substances telles que le cannabis, mais aussi la cocaïne ou l'héroïne.

Le premier concerne la présence d'une séquence temporelle : l'usage de tabac ou d'alcool précède en effet l'usage de cannabis dans la plupart des cas, ce qui semble en faveur d'une séquence où le tabac constitue la porte d'entrée vers le cannabis (Willner, 2001; Guxens et al., 2007a ; Guxens et al., 2007b). Le deuxième argument est constitué par la force de l'association statistique entre usage de substances licites et usage de cannabis. Une méta analyse effectuée à partir de nombreuses études, jugées comme étant de haute qualité méthodologique, rapportait un risque 1,7 à 2,6 fois supérieur d'expérimenter le cannabis pour des sujets ayant précédemment utilisé le tabac ou l'alcool (Guxens et al., 2007a). Ces résultats sont en faveur de la théorie, mais ne la prouvent évidemment pas (Popper, 1953). La théorie de l'escalade n'est d'ailleurs pas corroborée dans tous les travaux. Une étude réalisée auprès d'individus de classes sociales défavorisées de la banlieue de New York ne retrouvait la séquence testée (usage/abus d'alcool $\rightarrow$ usage/abus de cannabis $\rightarrow$ usage/abus d'autres drogues illicites) que chez 33\% des usagers de drogues (Mackesi-Amitti et al., 1997). Certaines études démontrent en effet que des variations individuelles (susceptibilité génétique, traits de personnalité, comorbidités psychiatriques, etc.) ou environnementales (disponibilité des drogues, effets observés et dangerosité perçue des produits, facteurs familiaux, influence des pairs, etc.) étaient susceptibles d'expliquer pourquoi des adolescents, après avoir expérimenté une drogue, pouvaient évoluer vers d'autres forme d'usages ultérieurement (Robinson, 2004). ${ }^{11}$

À l'heure actuelle, la grande majorité des études tend à démontrer une telle escalade de consommation, et ce plus particulièrement du tabac au cannabis et la «Gateway 
theory » soutient que la consommation de tabac et d'alcool sont des comportements presque indispensables à l'usage subséquent de cannabis, et à la consommation d'autres drogues par la suite, cependant la théorie à elle seule semble incomplète puisque d'après d'autres hypothèses la consommation de cannabis tend également à être un facteur de risque important pour l'usage subséquent de tabac .

Certaines études longitudinales ont en effet établi que la consommation de cannabis pendant l'adolescence est associée à un risque plus élevé d'initiation du tabac et à une future dépendance à la nicotine chez les jeunes adultes ,Parmi ces études, une étude australienne a indiqué que chez des adolescents non-fumeurs, l'usage hebdomadaire de cannabis amène plus de huit fois plus de risque d'initier la cigarette. De plus, pour les fumeurs de cigarettes ayant 21 ans, ce même niveau de consommation triple le risque de dépendance à la nicotine à l'âge de 24 ans. Une autre étude américaine réalisée chez des jeunes femmes âgées de 18 à 29 ans montre de son côté que celles ayant des antécédents de consommation de cannabis sont nettement plus susceptibles de commencer à fumer la cigarette régulièrement, et de devenir dépendantes à la nicotine tout récemment une autre étude longitudinale réalisée en Suisse a indiqué que, parmi les consommateurs de cannabis âgés de 16 ans ne fumant pas la cigarette, $28 \%$ ne fumeront que du tabac à l'âge de 22 ans, et 16\% deviendront consommateurs des deux produits $^{26}$. Globalement, les preuves scientifiques tendent maintenant à démontrer que la dépendance à la nicotine et la consommation persistante de cigarettes seraient les deux conséquences les plus néfastes de l'usage de cannabis pendant l'adolescence. ${ }^{26}$ puisque beaucoup de consommateurs de cannabis attestent que 1 ' associations entre cannabis et tabac ont même nui à leurs tentatives d'arrêt tabagique et que celui-ci les a poussé à fumer de la cigarette et n'a fait que renforcé la consommation de tabac s'ils en fumé auparavant.

Une autre théorie est venu contrecarrer la théorie de l'escalade stipulant que le passage d'une drogue à une autre ne serait à lui seule dépendre de la nature de la première substance utilisée, c'est la théorie des comportements à problèmes(Jessor \& Jessor, 1997) mais il dépend essentiellement des facteurs environnementaux du consommateur.Ces facteurs peuvent être liés au groupe de référence de l'individu(famille, pairs) ou liés à la communauté dans laquelle il évolue (école fréquentée, niveau de pauvreté, désorganisation sociale du quartier, etc.) tel qu'il a été rapporté précédemment ces facteurs sont le résultat d'une intrication complexe entre l'individu et son comportement addictif ,mais il reste tout de même que les deux théories se rejoignent sur un point commun qui est l'enchevêtrement de l'influence de l'environnement sur l'évolution de la consommation et la nature des substances utilisées .

Si les enquêtes en population générale adolescente offrent quelques pistes explicatives quant aux facteurs associés aux consommations régulières, le processus de construction d'habitudes de consommation demeure un point aveugle des connaissances actuelles. Ainsi, bien que les représentations et les motivations des usagers constituent un déterminant essentiel des conduites de consommation à l'adolescence, ces aspects restent encore peu appréhendés dans les études.$^{24}$ 


\section{6-Discussion}

Les recherches s'avèrent peu convaincantes quant aux trajectoires d'usage, de l'expérimentation à l'usage occasionnel chez les jeunes puis, le cas échéant, l'usage régulier voire quotidien. Au-delà de l'initiation, pourquoi certains jeunes poursuiventils leur consommation et pas d'autres ? Pourquoi certains d'entre eux s'installent-ils dans un usage régulier de tabac, d'alcool ou de cannabis, alors que d'autres s'arrêtent ou maintiennent leur consommation à un rythme occasionnel ?

Néanmoins deux théories ont essayé de mettre la lumière sur les liens causaux entre l'initiation précoce et les risques sanitaires et sociaux et peuvent être toutes deux vraies. La première est le «Gateway hypothesis » ou théorie de l'escalade selon laquelle la consommation précoce d'un produit addictif entraîne la consommation d'autres produits. Cette consommation précoce semble avoir un effet négatif sur la scolarité et, à plus long terme, sur l'insertion sociale et professionnelle La théorie de l'escalade a été mise en évidence et présume une relation causale de nombreuses études confirment cette hypothèse qui implique un enchaînement de nature causale avec toutes les réserves que ce terme peut soulever, malgré que celle-ci est de plus en plus remise en question l'association observée entre séquence et risque d'escalade des usages ne semble être que le reflet de facteurs en amont.

Par contre la seconde théorie est le «Common liability» ou théorie des comportements selon laquelle il existe une propension commune de développer des conduites addictives en lien avec des facteurs familiaux ou génétiques, et qui pourrait expliquer le lien entre une initiation précoce et l'addiction à plus long terme. ${ }^{16}$

Et que si la séquence d'usages de substances addictives tel que décrite par la théorie d'escalade (Gateway hypothesis) que nous avons observée semble réaliste et peut être étayée par de nombreuses explications. , une vulnérabilité commune aux addictions (Common liability model) pourrait expliquer aussi certaines pratiques addictives, l'hypothèse d'un facteur commun de vulnérabilité à différentes substances psychoactives pourrait constituer l'expression de facteurs individuels, notamment de la vulnérabilité génétique aux addictions (Gorwood et al. 2008). ${ }^{11}$

Et que selon cette hypothèse, l'ordre d'initiation des substances utilisées dépendrait de ces facteurs et non d'un ordre "préétabli" tel qu'il est postulé dans la théorie de l'escalade (Prince van Leeuwen et al, 2011).

Ces deux théories ont contribuées largement à l'explication des trajectoires et aux motivations de la consommation des produits psychoactives chez les jeunes et en particulier chez les adolescents, elles doivent plutôt être perçues comme complémentaires et ne sauraient expliquer à elles seules et indépendamment l'une de l'autre ce phénomène.

De par la gravité des conséquences relatives aux addictions installées (que ce soit à l'échelle individuelle ou collective), l'intérêt du dépistage précoce de ces conduites chez les individus à risque semble devenir de plus en plus primordial pour les chercheurs et les cliniciens, et doit être renforcé dans l'urgence par des stratégies de 
prévention à court et à long terme sur le terrain et auprès d'une population spécifique en quête d'aide et de soutien.

En matière de prévention auprès des adolescents consommateurs de tabac, il est important de tenir compte de plusieurs paramètres tels que l'âge et le sexe pour mieux consolider et installer une politique de prévention a adéquate et efficace, car les âges de 15 ans pour les garçons et de 17 ans pour les filles ne correspondent plus à ceux constatés depuis plusieurs années. L'adolescence est une étape marquée par l'augmentation de la taille et des masses musculaires, ainsi que par le développement des systèmes respiratoires et cardio-vasculaires. La maturation sexuelle caractérise la puberté, enfin, il se produit durant toute cette période un développement conjoint du cerveau, de l'intellect et du système méso-limbique dopaminergique qui sont le faisceau du plaisir et la récompense, sensible aux substances psychotropes et aux comportements à risques. La fin de l'adolescence est établie sur la consolidation des étapes du développement physique. ${ }^{1}$

Les stratégies de prévention du tabagisme doivent prendre en considération ces changements et ce processus de transformation afin de se positionner au mieux pour la prise en charge préventive de cette tranche, et tenir compte bien évidemment des mécanismes et des caractéristiques de la progression de l'usage du tabac. L'accent doit être mis sur les méthodes de résistance au stress et d'affirmation de soi, pour leur permettre de mieux se défendre dans les situations sociales où le risque de tabagisme est élevé. Pour ceux qui ont déjà commencé à fumer, à côté des actions générales indispensables comme la suppression des publicités et les interdits. ${ }^{25}$

L'intrication de plusieurs facteurs externes et internes de cette tranche d'âge peuvent être repérés dans le comportement du préadolescent tel que l'impulsivité, l'agressivité, la recherche de nouveauté, la désinhibition et les troubles du comportement. Ces facteurs sont parfois externalisés et deviennent un sujet d'inquiétude pour l'entourage. Il existe également des facteurs individuels plus internalisés, tels que l'affectivité négative qui entraîne la dépression, l'anxiété et le retrait social. Les 11-14 ans connaissent des difficultés d'autorégulation émotionnelle qui est un processus dynamique par lequel un individu mobilise ses ressources personnelles, sociales et environnementales. Ce processus permet à une personne de freiner ses comportements à risque, d'exercer un contrôle de son expression émotionnelle, de ses réponses affectives ainsi que d'anticiper les conséquences de ses actes. ${ }^{16 .}$

Toutefois, il ne faut pas négliger l'intrication entre les facteurs internes et les facteurs externes qui expliquent le degré d'influence des pairs chez les 11-14 ans. Un autre facteur environnemental est le facteur familial, l'attitude des parents à l'égard des substances est importante, de même que la qualité des relations positives entre les parents et le préadolescent protègent amplement. Un attachement fort à un modèle adulte estenlui meme un facteur de protection, de même que l'absence de troubles psychopathologiques, une bonne estime de soi, une capacité à choisir et à devenir autonome et des stratégies d'adaptation efficaces. Il s'agit d'identifier lesquels de ces facteurs de protection sont absents chez les individus les plus vulnérables. ${ }^{16}$ 
pour Bak \& Piko(,2007) il faut se placer dans une perspective développementale et adapter la prévention en fonction de la tranche d'âge des 11-14 ans, savoir repérer les idées négatives associées, car l'enjeu est de les conserver les plus longtemps possible dans ces représentations et les maintenir dans des attitudes d'abstinence au tabagisme, puisque certaines idées et représentations sur la dangerosité du tabac semblent protéger les jeunes de l'expérimentation et de l'installation dans l'habitude tabagique et la perception des risques liés au tabagisme diffère entre les jeunes et les adultes. chez ces derniers, un critère important dans l'attribution du risque est constitué par la quantité de cigarettes fumées. Le risque existerait seulement pour un nombre élevé de cigarettes certains estiment qu.il est très risqué de " fumer de temps en temps ", alors que d'autres jugent très risqué de " fumer un paquet ou plus par jour ». Les opinions sur les risques encourus évoluent très peu avec l'âge et ce, quel que soit le sexe ${ }^{15}$ donc la solution permettant d'éviter l'addiction au tabac c'est de ne pas commencer à fumer.

La susceptibilité de fumer est l'indice qui nous parait le plus adapté pour des population de jeunes enfants n'ayant jamais expérimenté le tabac, c'est les motifs pour lesquels il devient nécessaire de mettre en place une stratégie adaptée à cette tranche d'âge et l'école nous paritle lieu le plus favorable et le plus adéquat pour le repérage de ce genre d'expériences précoces, en somme il s'agit là d'intercepter les représentations et les motivations autour de l'usage du tabac du jeune enfant n'ayant pour la plus part jamais essayer de fumer Donc la prévention primaire nous semble la plus appropriée puisqu'elle consiste à programmer des interventions avant l'initiation et notamment dans les écoles primaires ou la prévalence de consommation de tabac est encore faible.

\section{Conclusion}

Il faut dire que le tabagisme constitue une des causes de morbidité et mortalité les plus évitables dans le monde, et c'est aussi la principale cause de mortalité précoce à l'échelle mondiale, en plus d'être un facteur de risque pour la consommation d'autres drogues, des problèmes graves de santé tels que les maladies cardiovasculaires, les maladies pulmonaires chroniques et le cancer du poumon y sont fortement liés, peutêtre extrêmement rares chez les jeunes, mais par contre la majorité des complications proviennent d'une consommation chronique ayant généralement débuté pendant l'adolescence, c'est en fait presque $90 \%$ des fumeurs adultes qui ont acquis cette habitude avant l'âge de 20 ans, donc toute consommation de tabac, mais surtout celle des adolescents, devrait ainsi être perçue comme inquiétante. ${ }^{26}$ Il est claire que ce soit pour ce mobile que la question de la précocité de la consommation de substances psychoactives mérite une attention particulière car c'est un facteur extrêmement prédictif de la survenue ultérieure d'un abus ou d'une dépendance. Sur ce plan, les stratégies préventives à mettre en œuvre doivent viser à retarder le plus possible l'âge de l'initiation aux consommations d'alcool, de tabac et de cannabis et ce, pour le plus grand nombre possible de pré-adolescents. Si la modification du cadre législatif (ou plus simplement une meilleure application des lois existantes) peut jouer un certain rôle en la matière, l'information et l'éducation à la santé doivent conserver une place majeure sur le volet des stratégies préventives. ${ }^{21}$ 
A présent qu' il est connu que c'est dès la préadolescence ou à un âge plus précoce que l'habitude tabagique s'installe et que probablement si ce n'est pas certainement, que d'autres habitudes addictives s'ensuivront.

Il est de prime à bord d'adapter des stratégies préventives afin d'arrêter la pandémie provoquée par le tabagisme qui consiste à arrêter le flux de nouveaux fumeurs dés le jeune âge, et éventuellement parer à tout risque d'addiction aux différentes sortes de substances psychoactives .

\section{Références bibliographiques}

1-Dreux Claude. la prévention en santé chez les adolescents, Groupe de travail Commission XVII, Institut national de santé publique, Québec. P6

2- La prévention du tabagisme chez les jeunes, (2004), avis scientifique, Institut national de santé publique, Québec, p12.13.14

3- Le cavalier, $\mathrm{M}$ et al, vers une compréhension du phénomène de dépendance, Centre DollardCormier, Institut Universitaire de dépendance, Québec. P14, 15

4- Tremey,A et al (2014), principaux risques liés à la consommation de substances psychoactives, Institut national de prévention et d'éducation pour la santé, France. P21

5- Tabagisme Prise en charge chez les étudiants, (2003) Expertise collective, INSERM, Les Editions INSERM14, 25,26

6- Gasman,I(2008) psychiatrie de l'adulte, de l'adolescent et de l'enfant, Editions VernazobresGreco,p145.14-

7- Conduites addictives chez les adolescent, (2014), Usages, prévention et accompagnement, expertise collective,INSERM ,France.p15,1

8- Kerjean(2005), Le tabac chez les adolescents. Comment les convaincre de ne pas fumer ? Comment les aider à arrêter de fume? Revue française d'allergologie et d'immunologie clinique, http//france.elsevier.com/direct/REVCLI, p562.

9- Kaoutar,K et al,(2008) , Tabagisme et facteurs associés des adolescents de la ville de Marrakech (Maroc), laboratoire d'écologie humaine, faculté des sciences semlalia, université cadi ayyad, marrakech,maroc.p98

10-Lafaye De Micheaux,C (2008), pratiques addictives à l'adolescence et théorie de l'attachement ,thèse pour le Doctorat en médecine, Faculté de médecine Xavier Bichat, université Paris 7 Denis Diderot.p, 8,9, 10 16,55,.

11- Mayet,A(2012), Etude des trajectoires d'usage de substances psychoactives, Thèse de doctorat en santé publique Spécialité épidémiologie , Université Paris V - Faculté de médecine Paris Descartes.p15,16,17 26,127, 128130

12-La consommation de drogues douces conduit aux drogues dures, www.lecavalierbleu.com/images/30/extrait 34.pdf.p3

13- Zertal Cherifa , Djenna,D, Kaabouche. A. Impact psychologique du tabac. Unité d'Aide Psychologique Chu Benbadis de Constantine. P3

14- Koenig,c.n et al, Tabagisme Les organisations responsables de la prévention \& Les moyens mis à disposition des fumeurs pour arrêter, Faculté de médecine ,Université de Genève .p7,

15-Cécile Bazillier,(2012),Etude longitudinale des effets de facteurs psychosociaux d'intervention de prévention sur la susceptibilité de fumer d'enfants de huit à onze ans ,thèse de Doctorat, Paris Ouest Nanterre. P21, 24,26, 43 
16- Varescon Isabelle, (2012), Drogues, alcool, tabac chez les 11-14 ans en savoir plus pour mieux prévenir, Auditorium Marceau, Paris. P 23, 30, 33,34.

17-Sofía Buelga et Gonzalo Musitu(2006), Famille et Adolescence. Prévention des Conduites à risque, Faculté de Psychologie, Département de Psychologie Sociale Université de Valencia. Espagne.p1

18- David Le Breton(2014), Adolescence et conduites à risque Edition Fabert .p14

19-Neurosciences : usage de substances psychoactives et dépendance Organisation Mondiale de la Sante' Genève. P13

20- JEUNES ET TABAC (2015), Etat de la situation. Cipret- vaud ligue de la santé . Suisse .p3

21-J. CARREAU(2003), Comportements de consommation de produits psychoactifs et dépendances, Résumé du rapport de santé, p1

22-Solange Carton.vulnérabilité au tabagisme .Laboratoire de psychologie clinique et de psychopathologie. Université René Descarte .Paris .p374

23-Befinoana, Noeline Razanamihaja(2011). tabagisme et facteurs associés chez les adolescents scolarisés à Madagascar. P466

24- Motivations et représentations associées aux usages de drogues chez les adolescents(2015) Projet d'étude ARAMIS (OFDT) , P 1,2, 3

25- Gilbert Lagrue. Le tabagisme des adolescents, un enjeu majeur en santé publique française, Centre de tabacologie, Hôpital Albert-Chenevier, Créteil .p155,156

26-François Bélanger et al (2010), Importance de l'exposition au tabac chez les jeunes consommateurs de cannabis un cheval de trois ?, Institut universitaire de médecine sociale et préventive, Lausanne. P4 